\title{
Patients as Teachers of Cultural Sensitivity in Pharmacy Education
}

Caitlin M Gibson, PharmD; Annesha White, PharmD, MS, PhD

University of North Texas System College of Pharmacy

\begin{abstract}
Introduction: Cultural sensitivity training among pharmacy students is required by the Accreditation Council for Pharmacy Education, but little data exists on effective practices for teaching these concepts. The goal of this case study was to describe the process and determine if integration of a patient-led Cultural Sensitivity Panel into the required didactic curriculum impacts pharmacy student perceptions of their own cultural competence.

Description of case: A special population was defined based on the CAPE competencies requiring students demonstrate sensitivity and responsiveness to culture, race/ethnicity, socioeconomic status, gender, sexual orientation, spirituality, disability, and other aspects of diversity and identity. Patients representing various special populations, such as veterans, the Deaf and hard of hearing population, the LGBT community, were invited to participate in a Cultural Sensitivity Panel for two consecutive years. Panelists shared information they wish future healthcare professionals understood about the population they represented and participated in a question and answer session. Pre-and post-surveys were conducted to assess the impact of the panel on student perceptions of cultural competence.

Results: Over two years, 138 students completed surveys. More than 95\% of students agreed or strongly agreed that a cultural sensitivity panel is a worthwhile experience, and that the panel would help them change behaviors that may be culturally insensitive. Student perceptions of their own cultural competence significantly improved between the pre-and post- surveys; ethnicity, age and gender significantly impacted responses ( $p<.05)$. Key themes of responses to open-ended questions included learning about effective communication (64\%), new resources for diverse patient populations (28\%), addressing barriers to care (21\%), the importance of patience and empathy (18\%), and incorporating a patient's background into their care (18\%).

Exploration of case impact: Use of a cultural sensitivity panel provides patients with their own voice in discussing barriers to the provision of health care and thus mitigates the inherent bias and limitations of faculty members teaching about cultures and populations they do not represent.

Conclusion: This novel approach of integrating a Cultural Sensitivity Panel into the didactic curriculum positively impacted student perceptions of their own cultural competence and may improve culturally competent provision of care among pharmacy students.
\end{abstract}

Keywords: Cultural Competency, Cultural Sensitivity, Pharmacy Education, Patient Panels

\section{INTRODUCTION}

The Office of Minority Health defines cultural sensitivity as "the ability to be appropriately responsive to the attitudes, feelings, or circumstances of groups of people that share a common and distinctive racial, national, religious, linguistic, or cultural heritage." ${ }^{1}$ The incorporation of cultural sensitivity training into the curriculum has been gaining attention across health care disciplines. The Accreditation Council for Pharmacy Education (ACPE) requires in the Center of the Advancement of Pharmaceutical Education (CAPE) outcomes that pharmacy curricula address cultural sensitivity and health care disparities. ${ }^{2}$ Although cultural sensitivity training was mandated by ACPE in 2006, ${ }^{3}$ and remains a key element of Standard 3 in the 2016 ACPE Accreditation Standards, Approach to Practice and Care, very few innovative methods to offer this training have been proposed in the last decade. The American College of Clinical Pharmacy (ACCP) established

Corresponding Author: Caitlin M Gibson, PharmD, BCPS, BCCP; Department of Pharmacotherapy, University of North Texas System College of Pharmacy, 3500 Camp Bowie Blvd, RES 411D, Fort Worth, TX 76107

Email: Caitlin.Gibson@unthsc.edu; Phone: (817) 735-0475 a Task Force on Cultural Competency, which published a series of White Papers from 2007 to 2013 that described the necessity of and best practices for culturally sensitive healthcare delivery and education. ${ }^{4-7}$ One paper in the series recommends that curricula incorporate culturally sensitive training focusing not only on race, ethnicity, and underserved populations, but also health disparities, social justice, disabilities, religion, and sexual orientation. ${ }^{6}$

\section{Impetus for Change}

The use of traditional teaching methods such as didactic classroom sessions utilizing slide set presentations and exambased assessments for cultural sensitivity training carries the risk of over-generalizations or stereotyping of patient populations. Additionally, faculty abilities to teach about patient groups they do not represent is limited. In this initiative, course faculty aimed to provide an opportunity for patients and their representatives to speak on their own behalf to articulate their experiences navigating the healthcare system. Out of this desire to allow patients to have a greater voice, a novel student-teacher relationship is proposed in this case study. This approach has two perceived advantages. First, faculty are removed from the frontlines of cultural sensitivity teaching, which may improve student buy-in into the concept. Second, this approach empowers the patient to speak up 
about their experiences and communicates to the students that patients are integral and crucial members of an effective healthcare team. A patient-led Cultural Sensitivity Panel provides an innovative teaching approach that may mitigate the teaching of stereotypes and promote cultural sensitivity as vital to positive patient outcomes.

\section{DESCRIPTION OF CASE}

The goal of this case study is to describe the process of incorporating patients as teachers of cultural sensitivity through a Cultural Sensitivity Panel, and to examine students' self-awareness of their level of cultural competence after exposure to the panel.

\section{Design}

The University of North Texas System College of Pharmacy (UNT SCP) is a recently established four-year pharmacy program in Fort Worth, TX. This study was conducted in PHAR 7263: Integrated Pharmacotherapy - Special Populations, a required component of the Spring curriculum for professional year three (P3) students, which is the final course in a foursemester Integrated Pharmacotherapy sequence. The course is designed to facilitate application of pharmacy knowledge to the care of diverse patients. Cultural sensitivity training is incorporated in teaching activities throughout the didactic curriculum, but PHAR 7263 houses the entirety of the contact hours dedicated specifically developing these skills.

The Cultural Sensitivity Panel has been a required component of the course since the course's inception in 2016. The panel was comprised of patients or representatives whose work intersects with various special populations. A special population in the course was defined based on the CAPE competencies requiring students demonstrate sensitivity and responsiveness to culture, race/ethnicity, socioeconomic status, gender, sexual orientation, spirituality, disability, and other aspects of diversity and identity. ${ }^{8,9}$ The course director used recommendations from the ACCP White Paper ${ }^{7}$ to identify potential special populations for representation on the panel and contacted various colleagues and community organizations to identify potential panelists who could represent these diverse patient populations. Those identified were selected based on their academic or practical expertise in the area of cultural sensitivity and were offered a $\$ 50$ stipend for their time. Table 1 lists a description of the panelists and the populations they represented.

Panelists were invited spend 5-8 minutes sharing knowledge, pearls, experiences, or attitudes they felt were most important for future health care professionals to learn about the population they represented. The panelist discussion was followed by an interactive question and answer ( $Q \& A)$ session between the students and panelists. In 2016, the panel was one hour long and five individuals agreed to participate. Based on student feedback in post-course evaluations, the panel was expanded to two hours in 2017; nine panelists agreed to attend but two reported on the day of the panel session that they would be unable to attend, resulting in a total of seven panelists. In addition to speaking and participating in the $Q \& A$ session, the panelists served several functions, including identification of additional members of the panel, the option to provide supplementary course materials relevant to this session (e.g. American Sign Language online dictionary, reference document of common terminology in the LGBT population), and communicating information about the initiative to their organizations. Panelists also wrote a short biography, which was distributed to the students one week before the panel session to allow students sufficient time to reflect on potential questions for the $Q \& A$ session.

\section{Evaluation of Student Learning}

Pre- and post-surveys were distributed to the students, with one week to complete. The pre-survey contained five Likertscale questions. The post-survey contained three sections. The first section contained structured questions used to gather data on the demographic characteristics and the cultural background of the respondents. Items included: gender, age, graduation year, and ethnicity. The second section included five 4-point Likert-scale questions (1=strongly agree, 2=agree, $3=$ disagree, and 4=strongly disagree) which are listed in Table 2. The final section contained two open-ended questions to gather feedback on using patients as teachers: 1) "Describe two things you learned during the cultural sensitivity panel and how they will impact the way you care for patients," and 2) "Do you have any feedback about what other types of patients or patient representatives should be included in the panel?" In 2017 the Likert scale question "I feel a cultural sensitivity panel is a worthwhile learning experience" also contained an openended response field in which students were asked to respond to the prompt "why or why not?"

Students were invited to complete the survey as part of their course participation. The essay questions were graded for completion by course faculty and comprised $2.5 \%$ of the student's final grade in 2016 and 5\% in 2017 to reflect the additional hour devoted to the experience. Adequate information, such as the aim of the research study, their expected participation, and their right to leave any part of the questionnaire unanswered if they felt uncomfortable answering it without any consequences to their part, were explained to the students. The cover page of the survey also provided the opportunity for students to opt-out of their data being used for research purposes.

This study employed a mixed-methods, descriptive, crosssectional design. Data collection was performed by the researchers in April 2016 and 2017. Data analyses were conducted using the SPSS version 23.0. An a priori level of $\alpha<.05$ was used to determine statistical significance. This study was approved by the University of North Texas Health Science Center Institutional Review Board. 
Open-ended essay responses from the question "describe two things you learned during the cultural sensitivity panel and how they will impact the way you care of patients" (Learning and Its Impact) were reviewed in aggregate and qualitatively analyzed manually to identify themes, according to the methods described by Castleberry and Nolen. ${ }^{10}$ First, responses to all essays were numbered sequentially and compiled into a single word processing document. The responses were then manually reviewed by one author to identify emerging commonly expressed concepts, ideas, and themes. A discussion of observed patterns was held among authors. The essays were then read again, and one or more descriptive codes were assigned to each response. The authors continued to meet to discuss findings to achieve a consensus. The descriptive codes were then reviewed and reassembled into overarching themes. A final round of discussion between authors was completed to finalize themes (Table 3). For example, the student comment "I thought it was really heartbreaking to hear how hard it is for this caretaker to look after her foster children in terms of getting insurance coverage for their medications" was coded as "empathy toward patient or caregiver," the comment "I learned about how diagrams can be used to convey medical information rather than words" was coded as "successful communication/translation services" and "I didn't realize there were so many resource options for the hearing impaired. I have a deaf patient at the pharmacy where I work [...] I don't know if he would be interested in using a translator/video but I like that I am able to talk to him about all of the options there are at his disposal" was coded as "familiarity with resources with which students were previously unfamiliar." All three descriptive codes fit under the broader theme of "successful communication/translation services."

\section{RESULTS}

A total of 69 students attended the Cultural Sensitivity Panel session in 2016 and 78 in 2017. Over the two years, a total of 137 students completed the pre-survey (93\% response rate) and 138 completed the post-survey ( $94 \%$ response rate). The mean age was 28 years and 55\% were female. Ninety-seven percent of students agreed or strongly agreed that the cultural sensitivity panel was a worthwhile experience, and $96 \%$ felt the panel would help them change behaviors. Table 2 lists the pre- and post- survey scores. Mean Likert scores improved between the pre- and post- surveys for all questions $(p<.05)$.

Open-ended reflections on Learning and Its Impact were completed by $93 \%$ of students and resulted in a total of 53 pages of text and almost 25,000 words. The key themes that emerged were learning about effective communication (64\%), new resources for diverse patient populations (28\%), addressing barriers to care (21\%), the importance of patience and empathy (18\%), and incorporating a patient's background into their care (18\%). A complete list of the most common themes is included in Table 3 and select quotes illustrating domain 3 learning are listed in Table 4.

\section{Identification of Case Themes}

Themes identified in the open-ended response questions were further explored. Student essays generally reflected either on the lessons communicated by one individual panelist, or tied together key talking points of multiple panelists into a broader "take home point." In addition, student responses could generally be categorized into specific, finite lessons likely to influence only a small number of patients in practice, or into broad, overarching lessons likely to influence a wide array of patient populations.

For example, when analyzing comments related to student learning, one commonly cited theme was learning about new resources to aid in patient care. The majority of these comments referred directly back to resources described by the panelist who works as an interpreter for Deaf and hard-ofhearing individuals. Students were encouraged to learn of various smartphone apps and online sign-language dictionaries that could be used in practice. While these resources will likely prove helpful when students care for these patients, the overall impact of these lessons may be limited by the relatively low proportion of the population who is Deaf or hard-of-hearing. In contrast, another common lesson learned related to displaying empathy towards patients based on the idea that one can never know what that individual has been through. Every single panelist was cited in essays related to empathy, indicating that this lesson is likely to touch a broad array of patients in practice and emerge as a key theme with regards to patient needs.

Similarly, when students were asked how the lessons they learned would influence their practice, some students shared specific and concrete examples, while others communicated the importance of general soft skills. For example, one student reflected on an anecdote shared by the Muslim refugee panelist. In this story, an English-speaking spouse misrepresented the health-related concerns of his wife who did not speak English because, in his view, she did not need medical care. The spouse's viewpoint was based on how healthcare was utilized in refugee camps mostly for emergencies, which in this case was different from the traditional American approach to preventative care. The student reported learning not to use family members of refugees for translation services. In contrast, another student reported on the overarching theme of incorporating an individual's background into their care and understanding what role family members play in the health of a patient. This student also reflected on the importance of using licensed translators and interpreters, but in a broader scope that implies an overarching lesson more likely to impact a larger group of patients.

The themes confirmed the findings of the Likert-scale questions. Students agreed with the statement "I know the meaning of the term 'cultural competency' significantly more after the patient panel experience. This change can be 
explained by lessons students learned related to behaviors which they may not have previously viewed as culturally insensitive. Additionally, students provided explicit examples of behaviors they planned to modify, confirming the change in the statement "I feel a cultural sensitivity panel will help me to change behaviors that may have been culturally insensitive."

\section{DISCUSSION}

Pharmacy students' self-awareness relates to their tendency to pay attention to their behavior, attitudes, and emotions in response to different situations. Gaining personal insight on one's own cultural biases is critical to the provision of quality patient centered care. As patient population demographics change with an increasing number of cultural perspectives, clinicians must become acquainted with a broad range of perspectives on health to keep pace with evolving patient demographics and students must refine their views and approaches to providing care. Provider-patient communication is linked to medication adherence, health outcomes, and patient satisfaction in receiving care. ${ }^{11}$ Therefore, students and educators must consider patient health perspectives influenced by gender, religion, ethnicity, race, language proficiency, and other cultural factors.

In this study, the incorporation of a new patient-led panel improved students' self-reported understanding of cultural sensitivity. Surveys indicated that upon completion of the session students not only gained a better understanding of cultural competence, but also intended to change behaviors related to cultural sensitivity. The key themes identified in the open-ended questions included specific tools students plan to implement, philosophical modifications in caring for patients, and intentionality in discovering and addressing how a patient's background may influence their care plan. Students were able to provide a range of specific examples of behavioral changes they planned to implement, suggesting that this activity may be the impetus for long-term, intentional development of cultural sensitivity skills among pharmacy students.

Our findings are consistent with previous research conducted by Poirier et al 2009, which showed improvements in selfreported scores measuring cultural sensitivity among pharmacy students enrolled in a three credit hour course. ${ }^{12}$ After employing a team-based learning approach to cultural sensitivity classroom training, approximately 70 pharmacy students were compared via pre- and post- course surveys. Students agreed that the cultural competence course enhanced their awareness of biases towards others $(p<.05)$ and authors concluded that cultural sensitivity was enhanced after the change in curriculum.

Studies show that cultural sensitivity training into the pharmacy curriculum through traditional lectures and patient cases is commonly used, but doesn't result in long term impact. ${ }^{13-15}$ In one study, provision of lectures and associated labs improved knowledge of health disparities and selfawareness related to cultural sensitivity, but inadequately equipped students with the knowledge and skills to address health disparities and deliver culturally competent care. ${ }^{13}$ Apart from classroom teaching, other activities cited in the literature include a poverty simulation ${ }^{16}$ and a six-week cultural sensitivity training series during Introductory Pharmacy Practice Experiences (IPPEs) ${ }^{17}$ which both improved outcomes related to cultural sensitivity for pharmacy students (e.g. importance of being aware of different cultures,) but their multimodal, longitudinal efforts fell short in reaching cultural competence goals as measured by the Inventory for Assessing Cultural Competence Among Healthcare Professionals-Revised (IAPCC-R) tool. ${ }^{14}$ During the training, faculty offered cultural sensitivity education using multiple methods in first, second, third and fourth year pharmacy courses (entitled Pharmacy Skills and Application series). A 25 item Inventory for Assessing the Process of Cultural Competence Among Healthcare Professionals - Revised (IAPCC-R) was used to assess cultural sensitivity. Authors noted wide variation in competence scores within each cohort and concluded that integrating cultural sensitivity content into their Pharmacy Skills and Application series was not sufficient to enhance students' cultural competence, highlighting the need for innovative approaches to teaching cultural sensitivity.

This study offers a unique approach to teaching cultural sensitivity by using patients as teachers. Although patients have been used in healthcare education for many years, the patient is often used as a case or a tool for presenting a specific medical condition, and the key learning points are communicated by a medical professional rather than the patient. As patient-centered care continues to be emphasized, there has been increased interest in utilizing the patient as an active teacher in medical education. Patient inclusion in education is an affirmation that patients are the experts on their own cultural and personal backgrounds which influence healthcare needs. ${ }^{18}$ The use of patients as teachers has been previously described in the context of teaching clinical skills, communication skills, ${ }^{18}$ and interactions with patients with disabilities. ${ }^{19}$ However, there is a paucity of literature on the use of patients as active teachers of pharmacy students. Moreover, there is little to no literature on how to best integrate patients into cultural sensitivity classroom training or the impact of using patients as teachers of cultural sensitivity.

\section{Exploration of Case Impact}

Faculty members must recognize their inherent biases which limit their ability to teach about cultures they do not represent. Use of a cultural sensitivity panel also provides patients with their own voice in discussing barriers to the provision of health care. Reduction of cultural sensitivity training to traditional lectures can result in stereotypes and generalizations and students memorize content of slides rather than work to achieve an overall understanding of cultural competence. Students expressed the importance of learning about cultures, 
but that individuals within a culture may still be vastly different. Students reflected on the importance of respect and compassion for all patients. Several essays discussed the importance of communication and incorporating an individual's beliefs into their health plan.

The positive results of the Likert-scale questions likely reflect the breadth and depth of lessons learned by students during the panel experience. Notably, there was a significant improvement in agreement with the question "I know the meaning of the term 'cultural competence' after the activity. In the open-ended section, students frequently described a behavior which they previously did not associate with cultural insensitivity, but the panelist testimonies increased the student's level of understanding of cultural competence through specific examples. In fact, statements including the sentiment "I did not know" occurred 58 times between the two reflection essays. Examples include "I did know [when communicating with a Deaf patient that] pronouncing words louder or talking slower changes the conformation of our mouth (sic) which makes it harder to read," "I did not know that autism manifests so differently in different individuals and so I will be careful in the future to ask permission before touching an autistic patient," and "I did not know that drugs go on and off [Medicaid] coverage so often and how challenging it is for parents to [...] get the medications they need for their loved ones."

Other colleges of pharmacy may consider incorporating similar panels into their curricula based on their individual needs. Colleges should to determine where gaps in cultural sensitivity training exist in the curriculum, and consider implementing a panel at that time. Because this session does not require basic science or pharmacotherapy knowledge, pharmacy students do not require any specific prerequisites for meaningful participation.

Panels in other cities and regions will naturally recruit participants more likely to be encountered in those regions and thus will organically meet the needs of pharmacy students in those areas. Based on our experiences, we believe that the exact "qualifications" of each patient to sit on the panel are less important than their ability to articulate their experiences within the healthcare system. For example, the critical themes that emerged from student reported learning included communication, empathy, withholding judgment, and incorporating a patient's background into their care. These themes are teachable by a wide variety of individuals, and the goal of the panel is to create key takeaway themes that will apply to a broader scope of patients rather than just the demographics and experiences of the specific patients on the panel. The open-ended questions were assigned to students to provide them a mechanism for reflection on their experiences with the panel. Targeted questions about lessons learned, when viewing the panel as a whole, encourage students to develop key takeaway messaging and consider how their lessons learned will be implemented throughout their careers. Future activities could include focus groups or application activities to help students translate the specific stories of panelists into long-term, actionable plans to continuously develop cultural competency skills.

The utilization of patients as teachers should not be restricted to pharmacy education. Because the accrediting bodies of several health disciplines incorporate cultural sensitivity training into core competencies, all health sciences disciplines have the potential to benefit from similar sessions. Faculty should consider using a patient panel as an interprofessional education (IPE) opportunity. As an example, colleges on our Health Science Center campus have shown interest in partnering and evolving the experience into an IPE experience complete with a post-panel case-based application exercise. Such a panel would achieve competencies for several healthcare disciplines, as well as the Interprofessional Education Collaborative (IPEC) competencies "embrace cultural diversity and individual differences characterizing patients, populations, and health care teams" and "develop a trusting relationship with patients, families, and other team members." ${ }^{20}$ Furthermore, the themes that emerged from this study were not pharmacy-specific. Indeed, several individuals from the Physician Assistant Studies program, who were members of LGBTQ student organization program at our health science center, attended the session as guests because of a relationship they had forged with one of our panelists. After the experience, the students expressed they had learned several useful lessons to apply to their future practices.

In addition to expanding the audience for the panel, educators can also build on to the panel with application activities. The use of patients as teachers lends itself to a discussion of implicit biases students may have related to individuals or groups represented on the panels. Cases could be useful application exercises for students to take the key themes and lessons from the panel and apply them to patient care. Students could discuss sensitive interviewing techniques, unique considerations for pharmacotherapy, or best practices for incorporating caregivers into a patient's care.

Robust assessment techniques to determine the long-term impact of patient panels should be created. The current activity was only assessed on a short-term basis using student perceptions. Follow-up assessments could examine student perceptions of the panel's value in fostering cultural sensitivity on experiential rotations. APPE rotation evaluations could include an assessment item allowing the preceptor to rate the student's cultural sensitivity.

Patients participating on the panel should have input on and evaluate the appropriateness of the environment to voice their lessons and the degree to which the relationship forged with the college was mutually beneficial. Faculty must be careful to ensure that patients are not made to feel like 
stereotypes or "token" representatives of a specific population. Patients must also feel free to honestly express their key takeaways from experiences with the healthcare system in a judgment-free environment. Absence of these conditions would be counterproductive to the mission of promoting true cultural sensitivity. Future studies should evaluate faculty participant perceptions, as well.

Although results were encouraging, this study is not without limitations. The two-year timeframe in which data was collected allowed an examination of themes from more than one cohort of students, however, the sample is confined to a single institution. Information about the students' prior diversity training, experience with patients with diverse racial or ethnic backgrounds taken care of in the past 12 months, home environment, and experiences with patients belonging to the special population groups encountered in the past 12 months was not collected. The survey tool utilized has not been previously validated. Additionally, the panelists were not formally surveyed on their perceptions and experiences within the panel. Finally, because of its cross-sectional design, this study does not assess the impact of the panel on cultural sensitivity outcomes during the Advanced Pharmacy Practice Experience (APPE) year or after graduation. This limitation is common to studies involving patients in education. One literature review identified 41 studies of patient involvement in education; only one study attempted to determine if the experience impacted everyday practice of the students. ${ }^{18-21}$

\section{CONCLUSIONS}

As the demographics of the United States continue to evolve, focus on culturally sensitive medical training and its impact on health outcomes is imperative. One method to teach cultural sensitivity is through the provision of a novel Cultural Sensitivity Panel focused on patients as teachers. It is important to note that all faculty and students must recognize their biases and limitations in teaching this content, which may lead to the reluctance for some programs to cover this material in their curriculum. Using patients as teachers offers an alternative and unique approach to gathering insights. Pharmacy curricula must keep pace with the evolving demographics in society by training students to provide patient-centered, culturally competent care. Future research is warranted to monitor long-term outcomes of such training.

\section{REFERENCES}

1. US Department of Health and Human Services. Office of Minority Health. National standards for culturally and linguistically appropriate services in health care.

https://minorityhealth.hhs.gov/assets/pdf/checked/ finalreport.pdf. Accessed 24 Nov 2018.

2. Accreditation Council for Pharmacy Education. Accreditation standards and key elements for the professional program in pharmacy leading to the doctor of pharmacy degre (Standards 2016). Approved January 25, 2015. http://www.acpeaccredit.org/pdf/Standards2016FINAL.pdf. Accessed 06 June 2019.

3. Accreditation Council for Pharmacy Education. Accreditation standards and guidelines for the professional program in pharmacy leading to the doctor of pharmacy degree, version 2.0. 2011;1-91.

Available from https://www.acpeaccredit.org/pdf/S2007Guidelines2.0 ChangesIdenti fiedInRed.pdf. Accessed 21 June 2018.

4. O'Connell MB, Korner EJ, Rickles NM, Sias JJ. Cultural competence in health care and its implications for pharmacy. Part 1. Overview of key concepts in multicultural health care. Pharmacotherapy. 2007;27(7):1062-1079. doi: 10.1592/phco.27.2.1062

5. American College of Clinical Pharmacy; O'Connell MB, Rickles NM, Sias JJ, Korner EJ. Cultural competency in health care and its implications for pharmacy. Part 2. Emphasis on pharmacy systems and practice. Pharmacotherapy. 2009;29(3):362. doi: 10.1592/phco.29.3.362

6. O'Connell MB, Rodriguez de Bittner M, Poirier T, Karaoui LR, Echeverri M, Chen AM, et al. Cultural competency in health care and its implications for pharmacy part 3A: emphasis on pharmacy education, curriculums, and future directions. Pharmacotherapy. 2013;33(12):e347-367. doi: 10.1002/phar.1353

7. O'Connell MB, Jackson AN, Karaoui LR, Rodriguez de Bittner M, Chen AM, Echeverri M, et al. Cultural competency in health care and its implications for pharmacy Part 3B: emphasis on pharmacy education policy, procedures, and climate. Pharmacotherapy. 2013;33(12):e368-381. doi: 10.1002/phar.1352

8. Medina MS, Plaza CM, Stowe CD, Robinson ET, DeLander G, Beck DE, et al. Center for the Advancement of Pharmacy Education 2013 educational outcomes. Am J Pharm Educ. 2013;77:Article 162. doi: 10.5688/ajpe778162

9. ACPE. Guidance for the accreditation standards and key elements for the professional program in pharmacy leading to the doctor of pharmacy degree. Guidance for Standards 2016. https://www.acpeaccredit.org/pdf/GuidanceforStandards2016FINAL.p df. Accessed 24 Nov 2018. 
10. Castleberry A, Nolen A. Thematic analysis of qualitative research: Is it as easy as it sounds? Curr Phar Teach Learn. 2018;10(6):807-15. doi: 10.1016/j.cptl.2018.03.019

11. Stewart MA. Effective physician-patient communication and health outcomes: a review. CMAJ. 1995;152(9):1423-1433.

12. Poirier TI, Butler LM, Devraj R, Gupchup GV, Santanello C, Lynch JC. A cultural competency course for pharmacy students. Am J Pharm Educ. 2009;73(5):Article 81. doi: 10.5688/aj730581

13. Okoro O, Odedina F, Smith WT. Determining the Sufficiency of Cultural Competence Instruction in Pharmacy School Curriculum. Am J Pharm Educ. 2015;79(4):Article 50. doi: 10.5688/ajpe79450

14. Haack S, Phillips C. Teaching cultural competency through a pharmacy skills and applications course series. Am J Pharm Educ. 2012;76(2):Article 27. doi: 10.5688/ajpe76227

15. Sales I, Jonkman L, Connor S, Hall D. A comparison of educational interventions to enhance cultural competency in pharmacy students. Am J Pharm Educ. 2013;77(4):Article 76. doi: 10.5688/ajpe77476

16. Clarke C, Sedlacek RK, Watson SB. Impact of a Simulation Exercise on Pharmacy Student Attitude toward Poverty. Am J Pharm Educ. 2016;80(2):Article 21. doi: $10.5688 /$ ajpe 80221
17. Vyas D, Caligiuri FJ. Reinforcing cultural competency concepts during introductory pharmacy practice experiences. Am J Pharm Educ. 2010;74(7):Article 129. doi: $10.5688 / a j 7407129$

18. Towle A, Bainbridge L, Godolphin W, Katz A, Kline C, Lown $B$, et al. Active patient involvement in the education of health professionals. Med Educ. 2010;44(1):64-74.

19. Jain S, Foster E, Biery N, Boyle V. Patients with disabilities as teachers. Fam Med. 2013;45(1):37-39. doi: 10.1111/j.1365-2923.2009.03530.x

20. Interprofessional Education collaborative Expert Panel. (2011). Core competencies for interprofessional collaborative practice: Report of an expert panel. Washington, D.C.: Interprofessional Education Collaborative. https://www.aacom.org/docs/defaultsource/insideome/ccrpt05-1011.pdf?sfvrsn=77937f97 2. Accessed August 15, 2019.

21. Thompson BM, Haidet $P$, Casanova R, Vivo RP, Gomez AG, Brown AF, et al. Medical students' perceptions of their teachers' and their own cultural competency: Implications for education. J Gen Intern Med. 2010;25 Suppl 2:S91-94. doi: 10.1007/s11606009-1245-9 
Table 1. Cultural Sensitivity Panelists

\begin{tabular}{|c|c|c|}
\hline Panelist & Population Represented & Years Present \\
\hline Military veteran* & US military veterans, Veterans Affairs healthcare & 2016, 2017 \\
\hline $\begin{array}{l}\text { Certified American Sign Language Interpreter } \\
\text { and sibling of Deaf individual }\end{array}$ & Deaf and hard of hearing & 2016, 2017 \\
\hline Adoptive mother of autistic children & $\begin{array}{l}\text { Adoption; foster system; caretaker for people } \\
\text { living with disabilities }\end{array}$ & 2016, 2017 \\
\hline Gay man and local LGBT advocate & LGBT community & 2016, 2017 \\
\hline $\begin{array}{l}\text { Hispanic clinic nurse (2016) or community } \\
\text { leader (2017) }\end{array}$ & $\begin{array}{l}\text { Hispanic community, English as a second } \\
\text { language }\end{array}$ & 2016, 2017 \\
\hline Laotian immigrant & $\begin{array}{l}\text { Immigrant community, English as a second } \\
\text { language }\end{array}$ & 2017 \\
\hline Bhutanese refugee & $\begin{array}{l}\text { Refugees, immigrants, English as a second } \\
\text { language }\end{array}$ & 2017 \\
\hline Lesbian woman and adoptive mother & Adoption, induced lactation, LGBT community & Unable to attend \\
\hline Muslim physician & $\begin{array}{l}\text { Muslim population, healthcare for Muslim } \\
\text { patients }\end{array}$ & Unable to attend \\
\hline
\end{tabular}

Table 2. Students' Pre- and Post- Test Cultural Sensitivity Panel Perceptions

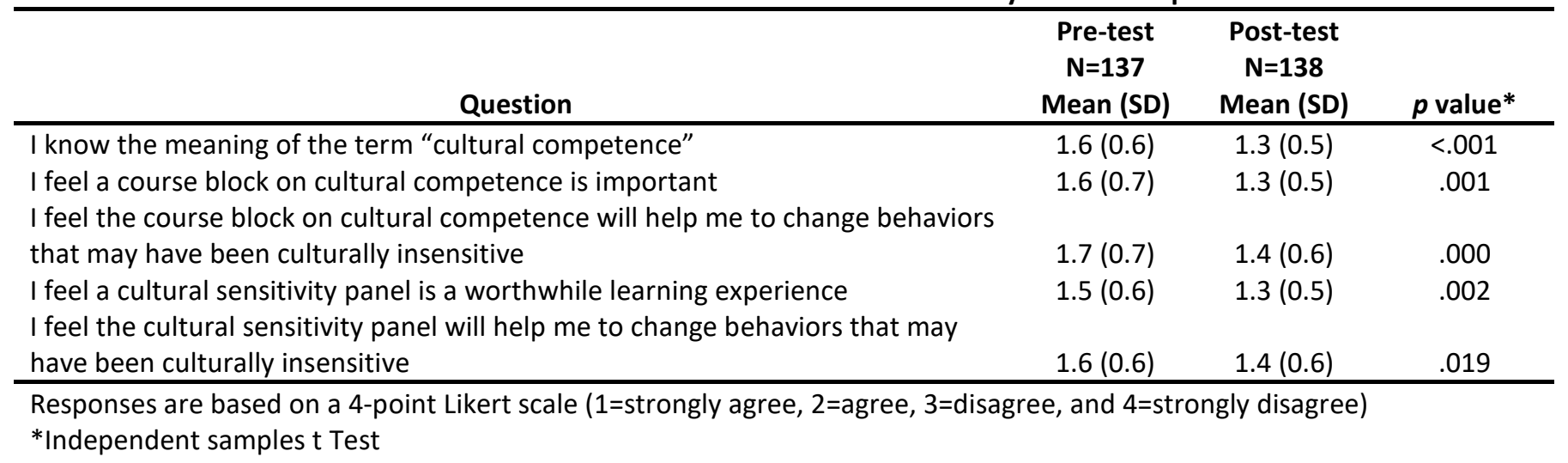

Table 3. Student Pharmacists' Learning and Its Impact Themes Post- Cultural Sensitivity Panel (n=138)

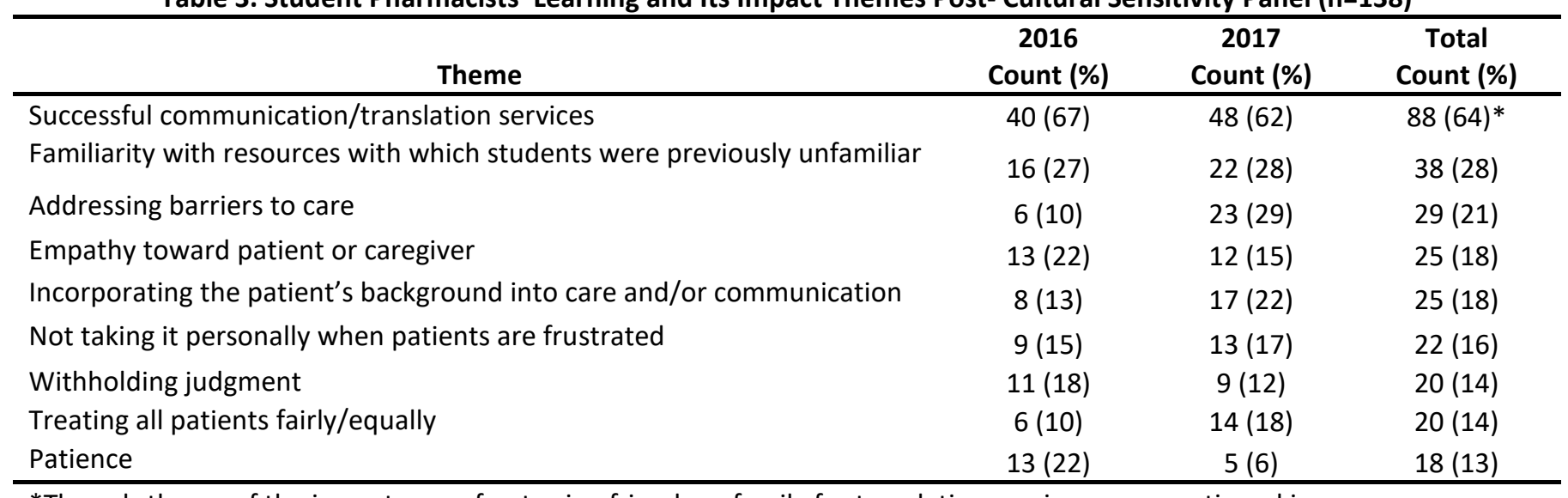

*The sub-theme of the importance of not using friends or family for translation services was mentioned in seven responses. 
Table 4. Select Quotes from Open-Ended Essays Pertaining to CAPE Outcome 3

\section{CAPE outcome}

3.5.1: Recognize the collective identity and norms of different cultures without overgeneralizing (i.e., recognize and avoid biases and stereotyping).

\subsection{2: Demonstrate an} attitude that is respectful of different cultures

\subsection{3: Assess a patient's} health literacy and modify communication strategies to meet the patient's needs.

\subsection{4: Safely and} appropriately incorporate patients' cultural beliefs and practices into health and wellness care plans.
"In society, it is easy to categorize someone who offends you by with a label. For example: That person with piercings giving you an attitude? Perhaps now you think all those with piercings have that kind of attitude. You quickly learn how negative this can become when someone who doesn't even know you begins to make assumptions just because of the kind of culture they think you might be. I believe it is important that the cultural sensitivity panel take place because it helps us build not only on cultural competence but on cultural compassion as well."

"During the cultural competency panel, I learned that while individuals may belong to or identify with the same cultural group, there can be differences within the group; not every experience is the same for similar individuals."

"I really valued [the veteran panelist's] advice about how to build rapport with veterans. He explained that veterans respond well to camaraderie, and that providing care in a way that promotes this will help gain patient trust and promote adherence."

"This panel reminds us of the humility we should have when working as a health care provider, not to make assumptions on other people's lives as we do not know their struggles, and to not to jump to conclusions just because someone may not be the same as us."

"Listening to the panel made me rethink how I have acted and treated those that don't share a culture with me. It is not often that I can sit and hear the perspective of other cultures. Everyone perceives things differently depending on their past experiences, [...] what their culture is, etc."

"I learned that I should not shy away from patients I may not be able to communicate to and should actually approach them and use whatever tools we were taught [about] to give them the best care."

"One must develop the necessary skills to communicate respectfully and sincerely in order to develop rapport with patients from different cultural backgrounds"

"Prior to the panel [...] I was unaware of the lack of treatment provided in refugee camps and did not understand the mistrust that some have towards the medical professional because of those experiences. It's helpful to know of these barriers to better address patients when providing counseling."

"One of the best ways to avoid putting yourself in uncomfortable or difficult situations is to prepare for them ahead of time. Being able to talk about the most common barriers across diverse demographics will help us as students be more prepared for the real life scenarios we will inevitably be a part of. Understanding viable options to help serve all people equally is a valuable lesson we can learn."

"Adherence goes way beyond the affordability, ease-of-use, and adverse event profile. Most of the time, we are trained as pharmacists to be advocates of medication adherence, using medication and lifestyle modifications as the drivers of success. However, some cultures do not embrace medicine as the primary tool to healing; instead, some ultimately believe in things like higher power and spiritual guidance in order to achieve health. In the future, I will address aspects like these and assess their importance in the care of my patients to promote a wellencompassing, patient-centered care model of pharmacy." 